\title{
DEEP INELASTIC SCATTERING
}


This page intentionally left blank 


\title{
DEEP INELASTIC SCATTERING
}

Proceedings of the 14th International Workshop

Tsukuba, Japan $\quad 20-24$ April 2006

\author{
editors \\ Masahiro Kuze \\ Tokyo Institute of Technology, Japan
}

\section{Kunihiro Nagano \& Katsuo Tokushuku}

High Energy Accelerator Research Organization (KEK), Japan

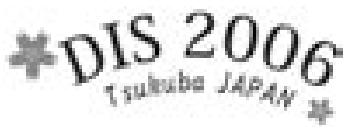




\section{Published by}

World Scientific Publishing Co. Pte. Ltd.

5 Toh Tuck Link, Singapore 596224

USA office: 27 Warren Street, Suite 401-402, Hackensack, NJ 07601

UK office: 57 Shelton Street, Covent Garden, London WC2H 9HE

\section{British Library Cataloguing-in-Publication Data}

A catalogue record for this book is available from the British Library.

\section{DEEP INELASTIC SCATTERING \\ DIS 2006}

Proceedings of the 14th International Workshop

Copyright $\odot 2007$ by World Scientific Publishing Co. Pte. Ltd.

All rights reserved. This book, or parts thereof, may not be reproduced in any form or by any means, electronic or mechanical, including photocopying, recording or any information storage and retrieval system now known or to be invented, without written permission from the Publisher.

For photocopying of material in this volume, please pay a copying fee through the Copyright Clearance Center, Inc., 222 Rosewood Drive, Danvers, MA 01923, USA. In this case permission to photocopy is not required from the publisher.

ISBN-13 978-981-256-871-7

ISBN-10 981-256-871-9 
This page intentionally left blank 


\section{Preface}

The Fourteenth International Workshop on Deep Inelastic Scattering and QCD (DIS2006) was held from April 20-24, 2006, at the International Congress Center EPOCHAL Tsukuba, in Tsukuba-city, Japan.

The annual DIS workshop was started in 1992 at Durham, in the same year that $\mathrm{H} 1$ and ZEUS began collecting data at HERA. Since then, the workshops have been held in Eilat, Paris, Rome, Chicago, Brussels, Zeuthen, Liverpool, Bologna, Cracow, St. Petersburg, Strbske Pleso and in Madison in 2005. The workshop in 2006 was the first time it was held in the Far East. About 240 experimental and theoretical physicists participated in the meeting, and many fruitful discussions ensued.

The main subject of the workshop has always been HERA physics. With time, however, more and more contributions have come from other experiments at Fermilab, SLAC, CERN, RHIC, Jefferson Lab and KEK, indicating the richness of the field of DIS. In the Tsukuba workshop, recent progress in the study of QCD from many approaches was vigorously discussed.

The HERA programme will finish in the summer of 2007, and the LHC at CERN will start in the near future. It was therefore timely that the workshop this year had a dedicated session to discuss the future directions for DIS physics.

The workshop was held with a support from Grant-in-Aid for Specially Promoted Research, from the Ministry of Education, Culture, Sports, Science and Technology (MEXT). The organizers wish to acknowledge financial support from Inoue Foundation of Science and from Tsukuba city. We also thank the High Energy Accelerator Research Organization (KEK) and Deutches Elektronen Synchrotoron (DESY) for financial assistance and invaluable help with the infrastructure and organization.

Proceedings Editors:

Masahiro Kuze (Tokyo Tech)

Kunihiro Nagano (KEK)

Katsuo Tokushuku (KEK) 
This page intentionally left blank 


\section{ORGANIZATION}

\section{Local Organizing Committee}

Ryosuke Hamatsu Tokyo Metropolitan Universty

Kaoru Hagiwara KEK

Jiro Kodaira KEK

Shunzo Kumano KEK

Masahiro Kuze Tokyo Institute of Technology

Kunihiro Nagano KEK

Naruhito Saito Kyoto University

Toshi-Aki Shibata Tokyo Institute of Technology

Katsuo Tokushuku KEK, Chairperson

Sakue Yamada KEK

Yuji Yamazaki KEK

\section{International Advisory Committee}

Giulio d'Agostini Roma

Guido Altarelli CERN

Violette Brisson Orsay

Dusan Bruncko Slovak Acad.

John Dainton The Cockcroft Institute

Andrzej Eskreys Cracow

Joel Feltesse Saclay

Rolf-Dieter Heuer DESY Hamburg

Robert Klanner DESY Hamburg

Max Klein DESY Zeuthen

Aharon Levy Tel Aviv

Lev Lipatov

Pierre Marage

St. Petersburg

Brussels

Rosario Nania Bologna

Jose Repond Argonne

Wesley Smith Wisconsin

Frank Sciulli Columbia

James Stirling Durham

Albrecht Wagner DESY Hamburg

Guenter Wolf DESY Hamburg 


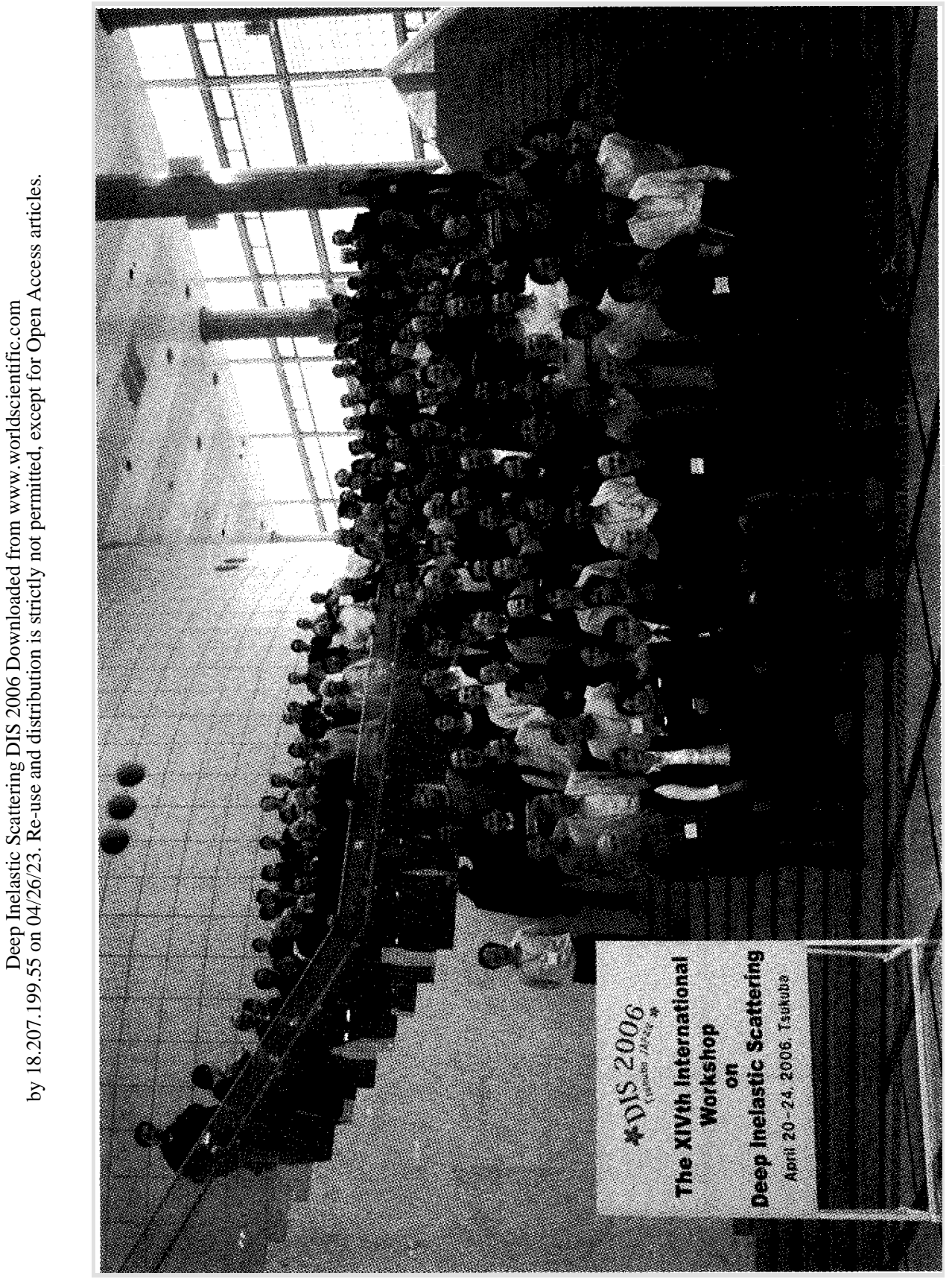




\section{CONTENTS}

Preface v v

Organization vii

Open Plenary Presentations

Recent Results from the H1 Experiment 3

E. Perez

Highlights from ZEUS $\quad 11$

M. Wing

Recent Results from the Tevatron

C. Royon

Results from the COMPASS Experiment at CERN

F. Kunne

Parton Distributions - DIS06

R. S. Thorne

Recent Developments in Perturbative QCD

G. P. Salam

In the Shadow of the Color Glass

E. Iancu

QCD and Monte Carlo Event Generators

Z. Nagy and D. E. Soper

\section{WG1: Structure Functions and Low- $x$ Working Group Presentations}

Recent Progress in Validation of the PDFs

S. I. Alekhin

Current Issues and Challenges in Global Analysis of Parton

Distributions

W.-K. Tung 
MRST Parton Distributions - Status 2006

R. S. Thorne, A. D. Martin and W. J. Stirling

Obtaining Parton Distribution Functions from Self-Organizing Maps

H. Honkanen, S. Liuti, Y. C. Loitiere, D. Brogan and P. Reynolds

From QCD at High Energy to Statistical Physics and Back

G. Soyez

Color Dipoles from Bremsstrahlung in High Energy Evolution

Y. Hatta

Impact Parameter Dependent Gluon Density from the

BK Equation

K. Kutak

How Resummation Depresses the Gluon at Small $x$

S. Forte, G. Altarelli and R. D. Ball

The DIS $(\chi)$ Scheme for Heavy Quark Production at Small $x$

C. D. White

Small- $x$ Resummation and Factorisation Schemes

D. Colferai

Prospects for the Constraining of PDFs from ATLAS Jet Data

D. Clements

Measurement of $F_{2}^{c \bar{c}}$ and $F_{2}^{b \bar{b}}$ at Low $Q^{2}$ and $x$ Using the H1 Vertex Detector at HERA

P. Laycock

Heavy Flavour Physics - FFNS and VFNS

R. S. Thorne

Heavy Quark Mass Effects and Heavy Flavor Parton Distributions W.-K. Tung

Neutral Current Cross Sections with Polarised Lepton Beam at ZEUS

S. U. Noor 
High $Q^{2}$ Neutral Currents in Polarised $e^{ \pm} p$ Collisions at HERA II 133 A. Nikiforov

Charged Current Cross Sections with Polarised Lepton Beams at ZEUS

H. Kaji

Charged Current Interactions in Electron-Proton Collisions at Hera II

B. Antunović

QCD and EW Analysis of the ZEUS NC/CC Inclusive and Jet Cross Sections

S. Shimizu

NNLO Analysis of Unpolarized DIS Structure Functions

J. Blümlein, H. Böttcher and A. Guffanti

Neural Network Determination of the Non-Singlet Quark Distribution

A. Piccione, L. Del Debbio, S. Forte, J. I. Latorre and J. Rojo

Bayesian Statistical Methods for Parton Analyses

G. Cowan

Progress on Global Analysis for Determining Parton Distribution Functions in Nuclei

M. Hirai, S. Kumano and T.-H. Nagai

Final Strange Asymmetry Results From NuTeV

D. Mason

$\mathrm{d}+\mathrm{Au}$ Collisions at STAR

C. A. Gagliardi

Precise Measurement of the Nuclear Dependence of Structure Functions in Light ${ }^{3} \mathrm{He}$ and ${ }^{4} \mathrm{He}$

J. Seely

DØ QCD Studies

$M$. Voutilainen 
xii

Constraints on PDF Uncertainties from CDF

C. Issever

Measurement of $F_{L}\left(x, Q^{2}\right)$ at HERA

R. S. Thorne

Prospects for a Measurement of $F_{L}$ at HERA with the ZEUS

Detector

D. Kollar

Future Measurement of $F_{L}$ with H1

M. Klein

Measurement of High- $x$ Neutral Current Cross Sections with the ZEUS Detector

A. Caldwell

\section{WG2: Diffraction and Vector Mesons Working Group Presentations}

Leading Baryon Production at ZEUS

203

M. Soares

Diffractive Cross Sections and Parton Densities from Rapidity

Gap and Leading Proton Measurements

P. Newman

H1 Diffractive Structure Functions Measurement from New Data

E. Sauvan

Theory of Diffractive Structure Functions

G. Watt, A. D. Martin and M. G. Ryskin

Diffractive Charm Production with the H1 Detector at HERA

o. Behnke

Diffractive Dijets and Combined Fits from H1

M. U. Mozer

Diffractive Production of Dijets and $D^{*}$ Mesons at ZEUS

A. Bonato 
Diffractive and Exclusive Measurements at CDF M. Gallinaro

Exclusive Vector Mesons at HERA

H. Kowalski

Diffractive Photoproduction of $\rho$ Mesons with Large

Momentum Transfer at HERA

C. B. Gwilliam

A New Measurement of Exclusive $\rho^{\circ}$ Photoproduction at HERA

J. Olsson

Deeply Virtual Compton Scattering at HERA II (H1 Results)

B. Roland

Ultraperipheral $J / \Psi$ and Di-Electron Production at RHIC (PHENIX)

M. Chiu

New Results on Spin Density Matrix Elements for $\rho^{0}$ at HERMES

B. Marianski

Diffractive $\rho^{0}$ Production at COMPASS

N. D'Hose

Prospects for Generalized Parton Distributions Studies at COMPASS

F, Heinsius

Review on Hard Exclusive Reactions

M. Diehl

The High-Energy Limit of Inclusive and Diffractive Deep

Inelastic Scattering in QCD

C. Marquet

Hard Diffractive Phenomena with Nuclei - from DGLAP to

Black Disk Limit and How They will be Studied in

Ultraperipheral Collisions at LHC

M. Strikman 
xiv

Transverse Imaging of the Proton in Exclusive Diffractive $p p$ Scattering

C. E. Hyde-Wright, L. Frankfurt, M. Strikman and C. Weiss

Diffractive Higgs Production at the LHC

A. D. Martin, V. A. Khoze and M. G. Ryskin

Hard Diffraction at the LHC

C. Royon

TOTEM: Early Diffractive Physics at the LHC

J. J. Whitmore

The FP $420 \mathrm{R} \& D$ Project at the LHC

B. E. Cox

Diffraction and Forward Physics at CMS

M. Ruspa

\section{WG3: Electroweak and Beyond the Standard Model Working Group Presentations}

Electroweak Measurements at the Tevatron

J. E. Garcia

Top Quark Properties at the Tevatron

P. Schieferdecker

Top Quark Pair Production Cross Section Measurements in $p \bar{p}$ Collisions at $\sqrt{s}=1.96 \mathrm{TeV}$

S. Cabrera

Top Physics at the LHC

M. P. Giordani

Events with High- $P_{T}$ Leptons and Missing Momentum at ZEUS M. Corradi

New H1 Results on Isolated Leptons and Missing $P_{T}$ at HERA

D. M. South 
Multi-Lepton Events and Doubly-Charged Higgs Production

at HERA

C. Vallee

Tau Production at HERA

333

S. Xella

$W$-Boson Production with Large Transverse Momentum at the LHC

N. Kidonakis, R. J. Gonsalves and A. S. Vera

High Energy Photon Interactions at the LHC

T. Pierzchala and K. Piotrzkowski

Search for Leptoquarks at HERA-I

C. Helebrant

Search for Excited Neutrinos at HERA

C. Diaconu

Solution to the LHC Inverse Problem

B. Knuteson

Non-SUSY Searches at the Tevatron

J. Kim

Higgs Searches at Tevatron

M. Tomoto

Supersymmetry Results at the Tevatron

F. Badaud

SPA Project: Supersymmetry Parameter Analysis at LHC/ILC

G. A. Blair, A. Freitas, H.-U. Martyn, G. Polesello W. Porod, M. Spira, D. Stöckinger and P. M. Zerwas

SUSY Searches with ZEUS

A. Montanari

LHC Prospects on Higgs Boson Searches

R. Ranieri 
$\mathrm{xvi}$

Measurement of the CKM Matrix Elements $\left|V_{c b}\right|$ and $\left|V_{u b}\right|$ at the B-Factories

W. Menges

Radiative and Leptonic Rare $B$ Decays from Belle

Y. Kwon

\section{WG4: Hadronic Final States Working Group Presentations}

Infrared Safe Definition of Jet Flavour

G. P. Salam

Heavy Quark Production and Non-Linear Gluon Evolution at the LHC

K. Peters

New Tests for the $k_{T}$-Factorization: Heavy Quark Production at HERA

A. V. Lipatov and N. P. Zotov

Studies of Top Pair Production in the Fully Hadronic Channel with CMS

C. Ciocca

Heavy Stable-Particle Production in NC DIS with the ZEUS

Detector

T. Matsumoto

Energy Flow Between Jets in the $k_{T}$ Algorithm

M. Dasgupta

Threshold Resummation for Higgs Production in Effective Field

Theory

F. Yuan

Measurement of Event Shape Variables in Deep-Inelastic

Scattering at HERA

J. Turnau

Event Shapes in Deep Inelastic Scattering at HERA

A. A. Savin 
M. Taševský

Prompt Photon Plus Jet Production with the ZEUS Detector

E. Brownson

Inclusive Prompt Photon Production in Deep Inelastic

Scattering at $\mathrm{H} 1$

C. Schmitz

Isolated Photon Cross Section Measurement at D $\varnothing$

A. Kumar

Scaled Charged Particle Momentum Distributions at High $Q^{2}$ at HERA

D. Traynor

Embedding NLO Calculations in Shower Event Generators with Positive Weights

P. Nason

Inclusive Hadron Electroproduction at HERA at NLO with and without Transverse-Momentum Constraint

B. A. Kniehl

Inclusive-Jet and Dijet Cross-Sections in High- $Q^{2}$ DIS at HERA

T. Schörner-Sadenius

Determination of the Strong Coupling Constant at LEP

T. Wengler

Soft-Gluon Corrections in Hard-Scattering Processes through NNNLO

N. Kidonakis

Inclusive Jet Production at the Tevatron

O. Norniella

Inclusive Jet Cross Section Measurement at $D \emptyset$ 
xviii

Jet Cross Sections in NC DIS and Determination of $\alpha_{s}$ at ZEUS

M. Jimenez

Inclusive Jet Production in Deep Inelastic Scattering at High $Q^{2}$ at HERA

S. Maxfield

Fast pQCD Calculations for PDF Fits

T. Kluge, K. Rabbertz and M. Wobisch

FastJet: Dispelling the $N^{3}$ Myth for the $k_{T}$ Jet-Finder

M. Cacciari

Numerical Evaluation of Loop Integrals

A. Daleo

Recent Progress in NLO Monte Carlos

S. Frixione

MadGraph/MadEvent - the New Web Generation

J. Alwall

THEPEG, HERWIG ++ and ARIADNE

L. Lönnblad

HELAC - A Monte Carlo Generator for Multi-Jet Processes

C. G. Papadopoulos and M. Worek

The Photoproduction Cross Sections of $\Lambda(1520)$ and $\bar{\Lambda}(1520)$

S. Wang

Measurement of $K_{S}^{0}, \Lambda$ and $\bar{\Lambda}$ Production at HERA

A. A. Savin

New Resonances and Spectroscopy

A. Kuzmin

Photoproduction of Events with Rapidity Gaps Between Jets at ZEUS

P. D. Ryan 
Multi-Jet Processes in the High Energy Limit of QCD

J. R. Andersen

Forward Jet Production In Deep Inelastic Scattering at HERA

C. Risler

Three-Jet Angular Correlations and Subjet Distributions at ZEUS

T. Schörner-Sadenius

Decorrelation of Dijets at Low $x$ and $Q^{2}$

M. Hansson

Parton Dynamics at Low $x$ Using DIS 3-Jet Events

C. Werner

Three- and Four-Jet Final States in Photoproduction at HERA

T. A. Namsoo

The Inclusive Jet Cross Section from STAR

M. L. Miller

Photoproduction of Dijets with High Transverse Momenta at HERA

K. Krüger

$W+$ Jet Production at CDF

A. Messina

Cross Sections at NNLO

V. Del Duca, G. Somogyi and Z. Trócsányi

\section{WG5: Heavy Flavour Working Group Presentations}

Experimental Results on Heavy Quark Fragmentation

L. K. Gladilin

$D$-Meson Production in the GM-VFN Scheme

B. A. Kniehl

Charmed-Hadron Production and Charm Fragmentation at ZEUS W. Dunne 
Charm Reconstruction in ZEUS HERA II Data

F. Karstens

Heavy Flavor Production in STAR

M. Calderón de la Barca Sánchez

Charm Production at Large Rapidities in $\mathrm{p}+\mathrm{p}$ and $\mathrm{d}+\mathrm{Au}$

Collisions at PHENIX at RHIC

X. Wang

Bottomium Production and $B_{s}^{0}$ Mixing at the Dø Experiment

T. Kuhl

Quarkonium Spectroscopy and New States from BaBar

L. Vitale

Heavy Flavour Production at HERA-B

R. Spighi

Elastic $J / \psi$ Production at HERA

Y. C. Zhu

Color-Evaporation Model vs. NRQCD in Charmonium Production G. T. Bodwin, E. Braaten and J. Lee

H1 Search for a Narrow Baryonic Resonance Decaying to $K_{S}^{0} p(\bar{p})$

D. Ozerov

Pentaquark Search at SPring-8 LEPS

N. Muramatsu

Pentaquarks - Some Theoretical Aspects

A. Hosaka

Probing QCD with Rare Charmless $B$ Decays

W. Gradl

Charm Baryons and Charmonium Production in $e^{+} e^{-}$Collisions at Belle

H. Kichimi

Charm Spectroscopy, Charm Decays and New States at BaBar M. Saleem 
$B$ Hadron Decays and Resonances at D0

D. Gelé

$b$-jets and $Z+b$-jets at $\mathrm{CDF}$

641

D. Jeans

Status of the Observed and Predicted $b \bar{b}$ Production at the

Tevatron

F. Happacher

Beauty Production with the ZEUS HERA II Data

O. M. Kind

Beauty Production Using $D^{*}+\mu$ and $\mu^{+} \mu^{-}$Correlations at ZEUS

A. E. Nuncio Quiroz

Measurement of Charm and Beauty Dijet Cross Sections in Photoproduction at HERA Using the H1 Vertex Detector L. Finke

Heavy Quark Production at HERA and its Relevance for the LHC 661 M. Wing

\section{WG6: Spin Physics Working Group Presentations}

Transversity Results from HERMES

L. L. Pappalardo

Transverse Quark Spin Effects in SIDIS and Unpolarized Drell Yan

L. P. Gamberg and G. R. Goldstein

Spin Dependent Fragmentation Functions at Belle

A. Ogawa, M. Grosse-Perdekamp and R. Seidl

Transverse Target-Spin Asymmetry Associated with DVCS on the Proton and a Resulting Model-Dependent Constraint on the Total Angular Momentum of Quarks in the Nucleon

Z. $Y e$

$A$ Polarization Measurements at COMPASS

B. Grube 
xxii

Spin Dependence in Polarized Elastic Scattering in the CNI Region 687 A. Bravar

Unifying the Mechanisms for Single Spin Asymmetries in Hard Processes

F. Yuan

Transverse Spin Physics at PHENIX

K. Tanida

Transverse Spin Studies with STAR at RHIC

C. A. Gagliardi

Single Spin Asymmetries of Identified Charged Hadrons in

Polarized $p+p$ at $\sqrt{s}=200 \mathrm{GeV}$

703

J. H. Lee

Universality of Single Spin Asymmetries in Hard Processes

P. J. Mulders, C. J. Bomhof and F. Pijlman

Proton Polarimetry at RHIC

A. Bravar

How To Extract $\Delta G$ from Measurements of $A_{\mathrm{LL}}$ ?

M. Stratmann

Transverse Double-Spin Asymmetries for Small $Q_{T}$ Drell-Yan Pair Production in $p p$ and $p \bar{p}$ Collisions

H. Kawamura, J. Kodaira and K. Tanaka

Threshold Resummation Effects in the Polarized Drell-Yan

Process at GSI and J-PARC

H. Yokoya and W. Vogelsang

Polarized Sea Measurements at JPARC

M. Contalbrigo, A. Drago and P. Lenisa

STAR Results on Longitudinal Spin Dynamics

J. Kiryluk

Overview of Longitudinal Spin Physics at PHENIX

M. X. Liu 
$\Delta G$ Measurements at PHENIX

Y. Fukao

$\Delta G$ from COMPASS

K. Kurek

STAR Spin Related Future Detector Upgrades

F. Simon

COMPASS Inclusive Asymmetries

751

M. Stolarski

Measurement of the Strange Quark Helicity Distribution from

Semi-Inclusive DIS on the Deuteron

755

M. Ehrenfried

Controversial Issues in the Polarized Parton Densities: $\Delta G, \Delta S$, Positivity and Higher Twist

E. Leader

Constraint on $\Delta G(x)$ at Large $x$

M. Hirai, S. Kumano and N. Saito

The Role of Semi-Inclusive Data in Polarized PDF Global Fits

G. A. Navarro and R. Sassot

Spin Structure Functions at Low $Q^{2}$ from JLab Data

771

K. V. Dharmawardane

Study of Duality in the Transition Region at JLab

Y. Prok

$Q_{T}$-Resummation for Polarized Semi-Inclusive Deep Inelastic

Scattering

Y. Koike, J. Nagashima and W. Vogelsang

The Resonance Spin Structure Measurement at Hall-C and the Future JLab Physics Program

S. Tajima 
xxiv

\section{Future of DIS Presentations}

eRHIC - A Precision Electron-Proton/Ion Collider Facility at Brookhaven National Laboratory

B. Surrow

J-PARC: Japan Proton Accelerator Research Complex

S. Sawada

The Future of DIS by Neutrino Beams

D. Mason

$F_{L}$ Measurements at HERA

A. Caldwell

High- $Q^{2}$ Physics at the LHC

F. Krauss

LHC Forward Physics

J. J. Whitmore

LHeC: The Large Hadron Electron Collider

J. Dainton

\section{Summary Presentations}

The Structure Functions and Low $x$ Working Group Summary

$V$. Chekelian, C. Gwenlan and R. S. Thorne

Summary of the "Diffraction and Vector Mesons" Working Group at DIS06

H. Lim, L. Schoeffel and M. Strikman

Electroweak Physics and Physics Beyond the Standard Model

L. Bellagamba, E. Sauvan and H. Spiesberger

Summary of the Hadronic Final State Working Group

T. Kluge, Z. Nagy and J. Terron

Summary of the Heavy Flavours Working Group

$U$. Karshon, I. Schienbein and P. Thompson 
